\title{
SISTEMÁTICA DEL GÉNERO BDALLOPHYTUM (CYTINACEAE)
}

\author{
Leonardo O. Alvarado-CÁrdenas
}

Universidad Nacional Autónoma de México, Instituto de Biología, Departamento de Botánica, 3er. circuito exterior s/n, Apdo. postal 70-367, Delegación Coyoacán, 04510 México, D.F. leonardo_ac@yahoo.com

\section{RESUMEN}

El género Bdallophytum está integrado por plantas parásitas de raíces y restringe su distribución a la parte septentrional del neotrópico. En México se presentan todas sus especies, dos de las cuales son endémicas. Anteriormente se le ubicaba en la tribu Cytineae de la familia Rafflesiaceae, pero según los estudios filogenéticos basados en datos moleculares, este grupo se reconoce ahora a nivel de familia como Cytinaceae. En su historia se han reconocido para Bdallophytum de uno a cuatro componentes y la última revisión taxonómica para el género fue realizada hace más de 70 años. El presente trabajo se apoya en un análisis de coordenadas principales para evaluar la identidad y número de miembros que lo integran, reconociéndose tres especies. Se proporciona una clave de su identificación, así como datos bibliográficos, descripciones morfológicas, enumeración de los ejemplares estudiados, información ecológica, mapas de distribución y fotografías.

Palabras clave: Bdallophytum, Cytinaceae, México, neotrópico, plantas parásitas.

\begin{abstract}
The genus Bdallophytum is comprised of root parasitic plants distributed in northern neotropics. In Mexico occur all the species of the genus, two of which are endemic. The genus has previously been positioned within the tribe Cytineae of the Rafflesiaceae, but based on results from molecular phylogenetic analyses, this tribe is currently recognized at the rank of family as Cytinaceae. Historically, one to four species have been accepted in the genus, and the most-recent taxonomic treatment for the genus was realized more than 70 years ago. In the present work, a principal coordinate analysis was conducted in order to evaluate the identity and number of species in this genus. The results of this analysis suggest
\end{abstract}


the recognition of three species. A taxonomic study with a key to species is presented, along with bibliographic information, morphological descriptions, specimens examined, ecological information, distributional maps and photos of the species.

Key words: Bdallophytum, Cytinaceae, Mexico, neotropics, parasitic plants.

\section{INTRODUCCIÓN}

El género Bdallophytum Eichler está integrado por plantas parásitas de raíces, principalmente de Bursera Jacq. ex L. (Burseraceae), que restringen su distribución al norte del neotrópico (Kuijt, 1969; Gómez, 1983). De acuerdo con los análisis filogenéticos basados en datos moleculares (Blarer et al., 2000; Nickrent et al., 2004), pertenece a la familia Cytinaceae, taxon segregado de Rafflesiaceae. El género ha sido abordado en diversas obras florísticas, aún como parte de la familia Rafflesiaceae, en las cuales se le ha considerado como integrado por una a cuatro especies (Harms, 1935; Gómez, 1983; Kuijt, 2001; Carranza-González, 2002). Aunque el conocimiento del grupo en general es escaso, cabe registrar algunos artículos que han indagado sobre diversos aspectos de la biología de una especie (García-Franco, 1996; García-Franco y Rico-Gray, 1997a, b; García-Franco et al., 1998), mismos que han brindado importante información sobre estas plantas. El último trabajo taxonómico que contempló a todos los componentes de Bdallophytum fue realizado hace ya más de 70 años (Harms, 1935). Por otra parte, el género está escasamente representado en los herbarios y, aunado a esto, muchos de los especímenes no se encuentran en buenas condiciones, lo que ha generado dudas acerca de la validez de algunos de los nombres propuestos. Bajo este panorama surgió la necesidad de evaluar la sistemática del grupo aplicando un análisis fenético, que permitiera explorar la identidad y el número de especies que le corresponden.

\section{MÉTODOS}

Se realizó una búsqueda bibliográfica relacionada con el género Bdallophytum y taxones afines que incluyó trabajos taxonómicos, morfológicos, ecológicos y filogenéticos: Solms-Laubach, 1901; Harms, 1935; Kuijt, 1969, 2001; Gentry, 1973; Gómez, 1983; Bouman y Meijer, 1986, 1994; García-Franco, 1996; García-Franco 
y Rico-Gray, 1997a, b; Meijer, 1997; García-Franco et al., 1998; Stevens, 2001; Carranza-González, 2002; Barkman et al., 2004; Nickrent et al., 2004; Burgoyne, 2006; Davis et al., 2007; Nickrent, 2007. Se revisaron ejemplares de los siguientes herbarios: ENCB, FCME, G, HUMO, K, MEXU, XAL y ZEA. La información fue complementada con la exploración en algunas bases de datos virtuales (Harvard University Herbarium (HUH), New York Botanical Garden (NY), Tropicos del Missouri Botanical Garden (MO) y United States National Herbarium (US)) y otros recursos electrónicos como The Parasitic Plant Connection y Angiosperm Phylogeny Website.

Para desarrollar el análisis fenético se creó una matriz de datos con un total de 23 caracteres morfológicos (Cuadro 1). La información se obtuvo de 31 ejemplares en buenas condiciones para evaluar sus características, los cuales están señalados con un asterisco $\left({ }^{*}\right)$ en el apartado de especímenes examinados del estudio taxonómico. También se integró la información de los ejemplares tipo de cada especie (ya sea a partir del protólogo y/o directamente del espécimen). La matriz básica de datos fue estandarizada empleando el promedio sobre la desviación estándar y sometida a un análisis de coordenadas principales (ACoP) utilizando el programa de NTSYSpc 2.11T (Rohlf, 2004). Los resultados se graficaron en dos dimensiones y a partir de ellos se realizó la definición taxonómica de las especies de esta manera reconocidas, acompañándolas de descripciones, claves de determinación, mapas de distribución y figuras para cada una.

Las características morfológicas a partir de las cuales fueron seleccionados algunos de los caracteres (ver Cuadro 1) para el análisis fenético se describen a continuación:

Hábito y estructuras vegetativas. Las especies de Bdallophytum son plantas parásitas sin clorofila (holoparásitas) que se alojan en las raíces de sus hospederos. Estas plantas han modificado su estructura vegetativa a un complejo celular llamado sistema endófito (Kuijt, 1969), el cual se desarrolla en todos los tejidos de la raíz, principalmente en el floema y xilema secundario del hospedero (GarcíaFranco, 1996). En la floración, el sistema endófito modifica la estructura del tejido hospedero, lo que le permite tener un abastecimiento constante de nutrientes en el desarrollo de la estructura floral, la cual emerge de raíces superficiales (GarcíaFranco, 1996).

Inflorescencias. Son de tipo espiciforme, con un eje floral bien desarrollado (carácter 1,2,3), de consistencia carnosa y cubierto con tricomas glandulares, los cuales pueden darle una coloración pardo-amarillenta. Las inflorescencias tienen escamas (carácter 4, 5) arrosetadas en la base del eje y dispuestas de forma alterna 
Cuadro 1. Lista de caracteres y estados de carácter empleados en el análisis fenético.

\begin{tabular}{|c|l|}
\hline Número & \multicolumn{1}{|c|}{ Carácter } \\
\hline 1 & longitud total de la inflorescencia $(\mathrm{cm})$ \\
\hline 2 & longitud del eje principal (cm) \\
\hline 3 & grosor del eje principal (mm) \\
\hline 4 & longitud de las escamas (mm) \\
\hline 5 & ancho de las escamas (mm) \\
\hline 6 & número de flores por inflorescencia \\
\hline 7 & tipo de flores (1- unisexuales, 2- hermafroditas) \\
\hline 8 & longitud de las brácteas (mm) \\
\hline 9 & ancho de las brácteas (mm) \\
\hline 10 & longitud del perianto (mm) \\
\hline 11 & diámetro de la base del perianto (mm) \\
\hline 12 & color del perianto (1- guinda, 2- morado) \\
\hline 13 & disposición de las anteras (1- anular, 2 -irregular) \\
\hline 14 & número de anteras \\
\hline 15 & longitud de los filamentos (mm) \\
\hline 16 & anteras con apéndices (1- si, 2- no) \\
\hline 17 & longitud de las anteras (mm) \\
\hline 18 & longitud del estilo (mm) \\
\hline 19 & número de placentas \\
\hline 20 & frutos concrescentes (1- si, 2- no) \\
\hline 21 & longitud del fruto (mm) \\
\hline 22 & ancho de los frutos (mm) \\
\hline 23 & longitud de las semillas (mm) \\
\hline & \\
\hline
\end{tabular}

a lo largo del mismo. Las escamas tienen margen serrado o entero, con una coloración morada a casi negra y están cubiertas por pubescencia glandular. El número de flores por inflorescencia (carácter 6) puede variar de acuerdo con la especie y es un carácter útil para el reconocimiento de las mismas.

Flores. Son epíginas, unisexuales o hermafroditas (carácter 7), dispuestas helicoidalmente en el eje, siempre bracteadas (carácter 8, 9). Presentan un olor fétido (Meijer, 1997; Steinmann 3080 (MEXU)), lo que junto con el color de la flor 
atrae moscas de los géneros Oxysarcodexia, Peckia y Perckiamya (García-Franco y Rico-Gray, 1997a, b). La envoltura floral no está diferenciada en cáliz y corola (carácter 10,11) y el número de segmentos es de $(4-) 8$ a 11, con longitud y forma irregular, y arreglo imbricado. El perianto es campanulado y está externamente cubierto por pubescencia glandular. La coloración varía de guinda a morada (carácter 12).

Androceo. Estructura taxonómicamente importante, pues permite la identificación rápida de las especies. Los estambres son monadelfos y los filamentos forman una columna (carácter 15), que en su parte apical está engrosada y sobre la cual se encuentran las anteras dispuestas de forma anular o irregular (carácter 13). Las anteras son oblongas en todas las especies pero pueden estar ligeramente curvadas o rectas, son de color blanco que contrasta con el del perianto; esta característica parece jugar un papel importante en la atracción de insectos polinizadores. Las anteras pueden tener una proyección del conectivo de color amarillento (carácter 16), que también posiblemente esté relacionada con la polinización, como sucede en otras familias; así por ejemplo, en Apocynaceae algunos de sus miembros cuentan con una proyección del conectivo (Endress, 1996).

Gineceo. El ovario es unilocular con siete o más placentas intrusivas (carácter 19), que portan un gran número de óvulos; éstos son ortótropos al igual que los de su grupo hermano Cytinus y unitégmicos (Bouman y Meijer, 1994). La individualidad del ovario puede ser muy evidente, como en Bdallophytum americanum, pero en las restantes especies el elevado número de gineceos cercanamente adyacentes no permite una clara separación de los mismos. El estilo es muy corto (carácter 18) con un estigma capitado muy notable, lobulado y de tono blanquecino a amarillento, que podría funcionar como un elemento atractivo para los polinizadores.

Fruto. Es una baya globosa (carácter 21, 22) densamente cubierta de tricomas glandulares. Las bayas en algunos casos se encuentran tan aglomeradas que llegan a formar una especie de fruto concrescente (carácter 20).

Semillas. Son numerosas por fruto y muy pequeñas (carácter 23), cubiertas de mucílago. La forma es globosa con una superficie ornamentada, carecen de rafe y el hilo y el micrópilo se encuentran en lados opuestos (Bouman y Meijer, 1994). Las semillas no presentan diferencias importantes que permitan separar a las especies. Los organismos registrados como dispersores son el roedor Peromyscus mexicanus mexicanus Saussure y hormigas del género Atta (García-Franco y Rico-Gray, 1997a, b). En el banco de semillas el germoplasma del parásito requiere de los estimulantes químicos del hospedero para su germinación (García-Franco y Rico-Gray, 1997a). 


\section{HISTORIA TAXONÓMICA}

La primera especie de Bdallophytum fue dada a conocer bajo el nombre de Cytinus americanus R. Br. (Brown, 1844), lo que representó el registro inicial en América de un género de holoparásitas hasta entonces conocido del Viejo Mundo. Posteriormente, Liebmann describió a Scytanthus Liebm. con una especie, $S$. bambusarum Liebm., pero no lo relacionó con el género Cytinus L. No obstante, Scytanthus no se acepta como nombre válido, ya que se le considera una variante ortográfica de un nombre más antiguo, Skytanthus Meyen, taxon perteneciente a la familia Apocynaceae (Harms, 1935). Años más tarde, Eichler (1872) propuso para la familia Balanophoraceae el género Bdallophytum con dos especies (B. andrieuxii Eichler y $B$. ceratantherum Eichler), sin tomar en cuenta las otras especies previamente descritas. Hacia finales del siglo XIX los taxones anteriormente descritos se integraron en un solo género, al cual los nombres de Bdallophytum (o su variante ortográfica Bdallophyton), Cytinus L., Hypocistis Adans. y Scytanthus Liebm. fueron aplicados de forma indistinta (Hooker, 1873; Hemsley, 1882; Kuntze, 1891; Solms-Laubach, 1889). No es sino ya en el siglo XX, cuando todas las especies americanas en cuestión fueron circunscritas dentro de Bdallophytum (Solms-Laubach, 1901; Harms, 1935). Este género y los restantes miembros de Cytinus se ubicaron dentro de la familia Rafflesiaceae, como la tribu Cytineae (Solms-Laubach, 1889; Harms, 1935). Desde entonces, la posición del grupo como tribu se ha mantenido por mucho tiempo, aunque algunos autores ya anticiparon su elevación a nivel de familia (Hutchinson, 1959; Takhtajan, 1997).

El empleo de marcadores moleculares del núcleo y de la mitocondria en análisis filogenéticos han revelado que la concepción de la familia Rafflesiaceae en el sentido tradicional, integrada por tres o cuatro tribus (Kuijt, 1969; Cronquist, 1981; Bouman y Meijer, 1986), es una propuesta artificial, basada en caracteres convergentes como endoparasitismo, reducción de estructuras vegetativas, flores endógenas y parecido en estructuras reproductivas. La evidencia filogenética revela que las tribus circunscritas en Rafflesiaceae tienen afinidades con conjuntos ubicados en órdenes distintos y han sido elevadas al nivel de familia. El trabajo de Nickrent et al. (2004) provee el primer panorama general de la relaciones de este grupo de parásitos y muestra que los integrantes de la familia Cytinaceae, antes la tribu Cytineae, se encuentran relacionados con taxones del orden Malvales, donde potencialmente están vinculados con otro conjunto también segregado de Rafflesiaceae (la familia Apodanthaceae), aunque las conexiones entre ambos grupos y la posición relativa de éstos aún no están resueltos (Nickrent et al., 2004). La 
evidencia recientemente obtenida coloca a Cytinaceae como hermano de la familia Muntingiaceae, que junto con miembros de Thymelaeaceae forman el grupo hermano del orden Malvales s. l. (Nickrent, 2007). Los restantes géneros de Rafflesiaceae (Rafflesia R. Br., Rhizanthes Dumort. y Sapria Griff.) están más relacionados con miembros del orden Malpighiales (Barkman et al., 2004; Davis et al., 2007). En el presente trabajo se considera que existen razones morfológicas y moleculares suficientes para reconocer a Cytinus y Bdallophytum como componentes de una familia distinta.

\section{RESULTADOS}

Análisis numérico

El análisis de coordenadas principales (ACoP) realizado para las especies de Bdallophytum indicó que los primeros dos componentes explican $83.6 \%$ de la variación. En la figura 1 se pueden reconocer tres grupos que fueron delimitados por elipses. El primer eje separa dos y el segundo complementa el reconocimiento de tres conjuntos que pueden considerarse como especies distintas. Los caracteres diferenciales más importantes fueron el número de flores (6), el tipo de flores (7), la longitud del perianto (10), el color del perianto (12), la disposición de las anteras (13) y la presencia de apéndices en las anteras (16).

Los especímenes tipo correspondientes a Liebmann 1898 (B. bambusarum), Barclays.n. (B. americanum) y Ervendberg 101 (B. ceratantherum) se agruparon en una de las nubes de puntos. El ejemplar de Andrieux 50 (B. andrieuxii) y el de Pringle 4373 (B. oxylepis) quedan incluidos en distintos conjuntos. El patrón resultante permitió reconocer a tres especies, las cuales se abordan a continuación.

Taxonomía

Aunque ya existen trabajos que tratan a las tribus de Rafflesiaceae como cuatro entidades distintas (Stevens, 2001; Burgoyne, 2006; Nickrent, 2007), en esta aportación se considera conveniente proporcionar una descripción de la familia Cytinaceae junto con los taxones aquí abordados.

Cytinaceae (Brongn.) A. Rich. in Bory, Dict. Class. Hist. Nat. 5: 301. 1824. Cytineae (Brongn.) Spach, Hist. Nat. Vég. 10: 551.1841 (Eucytineae). Cytinoideae Link, Handbuch 1: 368. 1829. TIPO: Cytinus L. (1764). 
Plantas holoparásitas, dioicas, monoicas o rara vez polígamo-monoicas, aclorófilas. Tejido vegetativo transformado en un sistema endófito y embebido en el hospedero. Escamas foliares arrosetadas en la base e imbricadas a lo largo del eje floral, simples. Inflorescencias en cimas, racimos o espigas, pero hacia el ápice con un patrón umbeliforme, o rara vez flores solitarias, bracteadas. Flores por lo general unisexuales, cuando son hermafroditas las anteras se encuentran opuestas al estigma, actinomorfas bracteadas; perianto tubular a campanulado, de color rosa, rojo, guinda, morado, amarillo o blanco; flores masculinas con perianto 4-9-lobulado, imbricado, nectario presente, en forma de pequeñas papilas

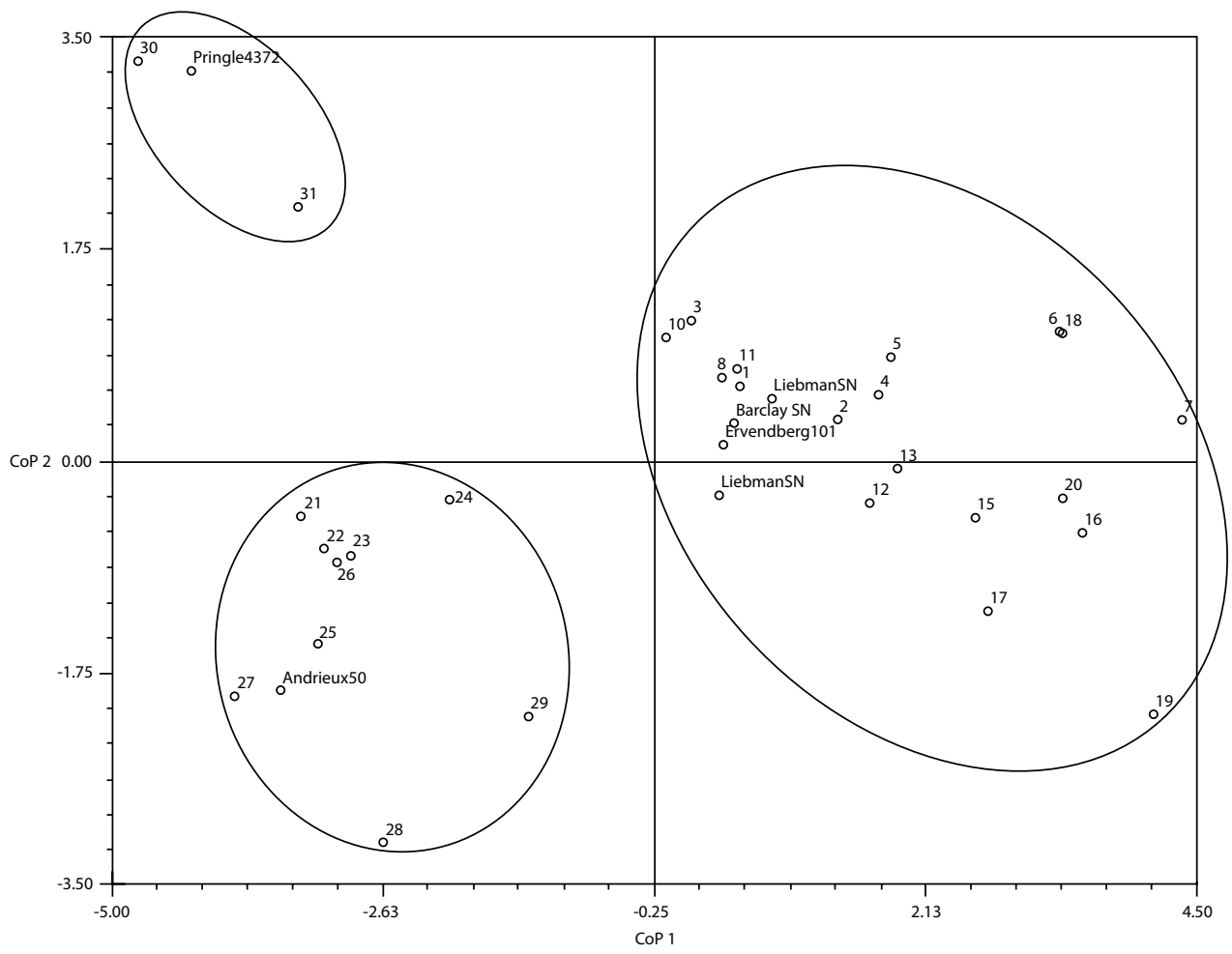

Fig. 1. Gráfica del análisis de coordenadas principales en el que se han delimitado con elipses tres agrupaciones, los cuales se interpretan como especies distintas. Los nombres en cada conjunto corresponden a los ejemplares tipo de las especies reconocidas para Bdallophytum: B. americanum (Barclay s.n.), B. andrieuxii (Andrieux 50), B. bambusarum (Liebmann s.n.), B. cerantatherum (Ervendberg 101), B. oxylepis (Pringle 4373). 
en la base del perianto, filamentos monadelfos, formando una columna, anteras (2-)5-14(-18), algunas veces con un apéndice estaminal, extrorsas, dehiscencia longitudinal; flores femeninas con un perianto más pequeño que en las masculinas, ovario 1-locular, ínfero o semiínfero, óvulos numerosos, placentas 8-14, parietales intrusivas, nectario presente como en las masculinas, estilo columnar, estigma capitado, lobulado, viscoso. Fruto una baya, a veces con dehiscencia irregular, pulpa mucilaginosa, traslúcida; semillas numerosas, muy pequeñas, globosas. Número cromosómico $n=12$ y 16 .

Diversidad y distribución. Familia con dos géneros, Cytinus ( 8 spp.) registrado de Europa y África y Bdallophytum (3 spp.) presente en México y Centroamérica.

Comentario. La cercana relación entre Cytinus y Bdallophytum fue reconocida desde el siglo XIX (Brown, 1844; Solms-Laubach, 1889); este supuesto no ha cambiado desde entonces y la hermandad entre estos taxones se ha corroborado con datos morfológicos y moleculares (Kuijt, 1969; Takhtajan, 1997; Bouman y Meijer, 1994; Nickrent et al., 2004; Nickrent, 2007).

Bdallophytum Eichler, Bot. Zeitung (Berlín) 30: 709. t. 8. 1872. Scytanthus Liebm., Förh. Skand. Naturf. Möte 4: 183. 1847; non Skytanthus Meyen 1834, nec W. J. Hooker 1844. Bdallophyton Eichler, in Engler \& Prantl, Nat. Pflanzenfam. 3(1): 281. 1889 var. orth. TIPO: Bdallophytum andrieuxii Eichler (aquí designado).

Parásitas dioicas o polígamo-monoicas con ejes florales bien desarrollados, carnosos, no ramificados, de color guinda a morado, esparcida a densamente pubescente-glandulares, loqueles da una coloración pardo-amarillenta. Inflorescencia espiciforme. Flores unisexuales o hermafroditas, perianto irregularmente segmentado, campanulado, de color guinda a morado, negro en una etapa más madura, externamente pubescente-glandular. Ovario globoso, con 7-12 placentas; estigma capitado, lobulado. Bayas globosas u ovoides, a veces concrescentes, densamente pubescente-glandulares; semillas con testa ornamentada, amarilla. Número cromosómico $n=12$ (en B. americanum).

Diversidad y distribución. Género endémico de América con tres especies que se distribuyen desde el centro de México hasta Costa Rica. En México existen todas, dos de ellas son endémicas. 
Comentarios. En la descripción original del género, Eichler (1872) reconoció a dos especies: $B$. andrieuxii y $B$. ceratantherum, sin definir el tipo. En este trabajo se designa a $B$. andrieuxii como la especie tipo, ya que $B$. ceratantherum se considera ahora sinónimo de $B$. americanum.

Bdallophytum se distingue de Cytinus por su eje floral espiciforme no ramificado (Cytinus tiene flores solitarias o inflorescencias en racimos o glomérulos), las flores guindas o moradas (contra amarillas, rojas y blancas) y las semillas ornamentadas (contra lisas); la distribución de ambos grupos no se traslapa.

Los ejemplares de este género son en ocasiones mal determinados como miembros de Orobanchaceae o Lennoaceae por su hábito holoparásito, los tallos carnosos y las flores moradas. Sin embargo, ambas familias son fácilmente diferenciables de Bdallophytum por sus flores pediceladas, corolas tubulares o bilabiadas, estambres libres, ovario súpero y frutos capsulares.

Clave para las especies

1. Inflorescencias con menos de 25 flores; anteras generalmente rectas, conectivo apical acuminado; bayas globosas, no concrescentes ............. B. americanum

1. Inflorescencias con más de 40 flores; anteras curvadas, conectivo apical ausente; bayas ovoides, concrescentes.

2. Plantas dioicas; anteras 8-14(-18), dispuestas de forma irregular en el ápice de la columna estaminal B. andrieuxii

2. Plantas polígamo-monoicas; anteras 4-8, en las flores hermafroditas éstas se encuentran opuestas al estigma en el ápice de la columna estaminal .....

B. oxylepis

Bdallophytum americanum (R. Br.) Eichler ex Solms in Engler \& Prantl, Nat. Pflanzenfam 3(1): 282. 1889; non Bdallophyton americanum (R. Br.) Harms in Engler \& Prantl, Nat. Pflanzenfam. ed. 2, 16b: 281; 1935 nom. illeg. Cytinus americanus R. Br., Trans. Linn. Soc. 19: 246. 1844. Cytinus americanus Hook. f. in DC. Prodr. 17: 108. 1873. pro parte; Scytanthus americanus (R. Br.) Solms in Engler, Pflanzenr. IV. 75(Heft 5): 17. 1901. TIPO: “America aequinoctialis”, s.d., A. Barclay s.n. (holotipo BM) (Fig. 2).

Scytanthus bambusarum Liebm., Förh. Skand. Naturf. Möte 4: 177. 1847; Bdallophyton bambusarum (Liebm.) Harms in Engler \& Prantl, Nat. Pflanzenfam. ed. 2, 

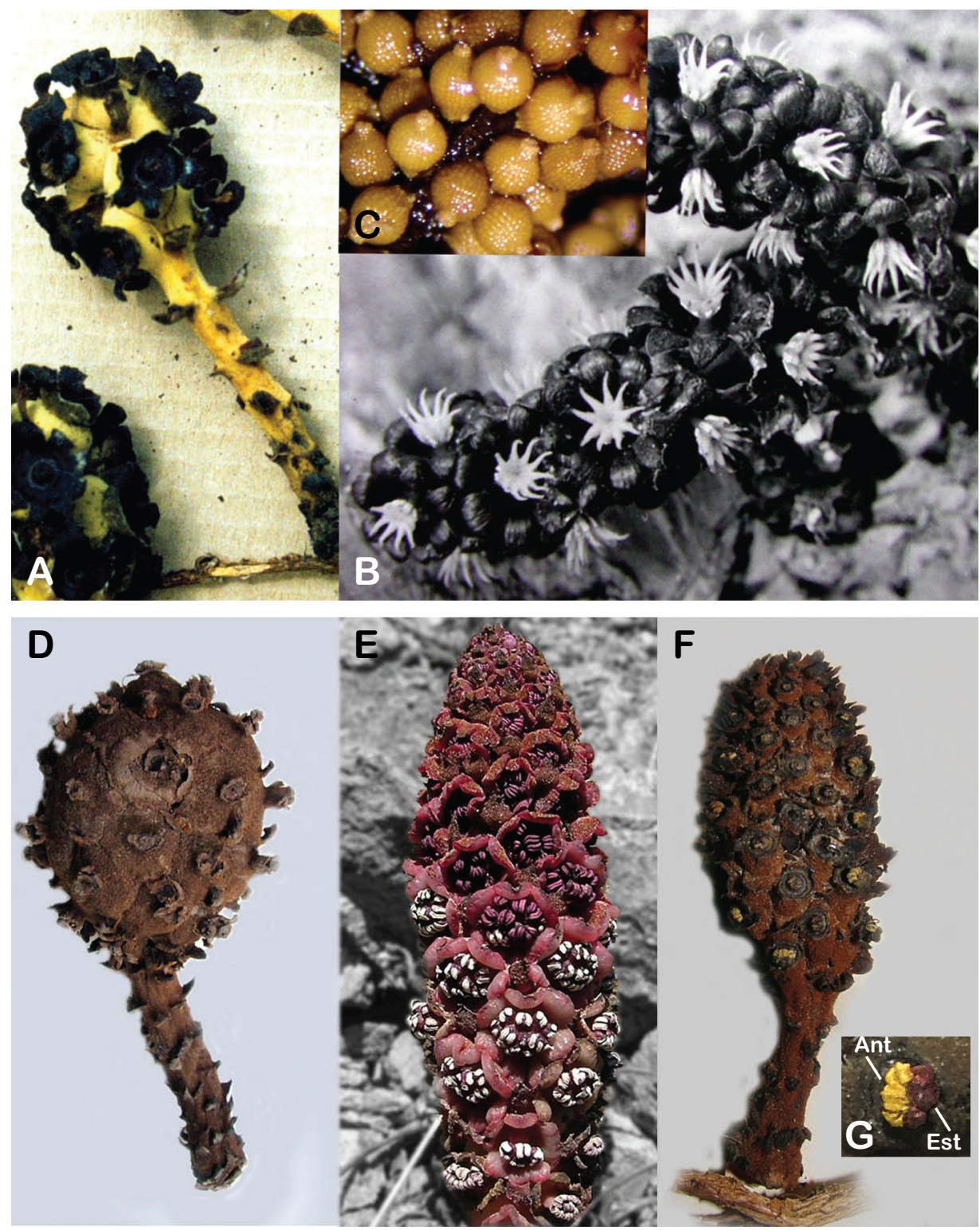

Fig. 2. Especies del género Bdallophytum. A-C. B. americanum: A.- individuo femenino (Foto de E. Carranza), B. individuo masculino, C. semillas. D-E. B. andrieuxii: D. individuo femenino, E. individuo masculino. F-G. B. oxylepis: F. individuo polígamo-monoico, G. detalle de la flor hermafrodita (Ant: antera; Est: estigma). 
16b: 281. 1935. TIPO: (México. Veracruz): Inter Paso del correo et Papantla, Jun 1841, F. M. Liebmann 1989 (holotipo C; isotipo GH).

Bdallophytum ceratantherum Eichler, Bot. Zeitung (Berlín) 30: 715. 1872, TIPO: México. Bei Wartenberg in der Nahe von Tantoyuca, mexicanische provinz Huazteca, 1858, L. C. Ervendberg 101 (holotipo no localizado en Herb. Boissier, probablemente en B (destruido) o M).

Hierbas 8-15.8(-25) cm alto, dioicas; escamas 0.5-1.5 x (3.4-)4.5-6.3 mm, ovado-lanceoladas, elípticas a espatuladas, margen irregularmente serrado o entero, carnosas, pardas, pubescente-glandulares. Inflorescencias con menos de 25 flores. Flores masculinas con brácteas 3.3-6.7 x 4-6 mm, ovadas, lanceoladas o espatuladas, margen serrado, pardo-rojizas a negras; perianto 6-9-segmentado, 6.5-10.9 mm alto, 5-6.4 mm diámetro en la base, de color guinda a negro; columna estaminal 1.4-2.7(-3.5) mm largo, anteras (6-)8-10(-11), 2.7-3.3 mm largo, generalmente rectas,

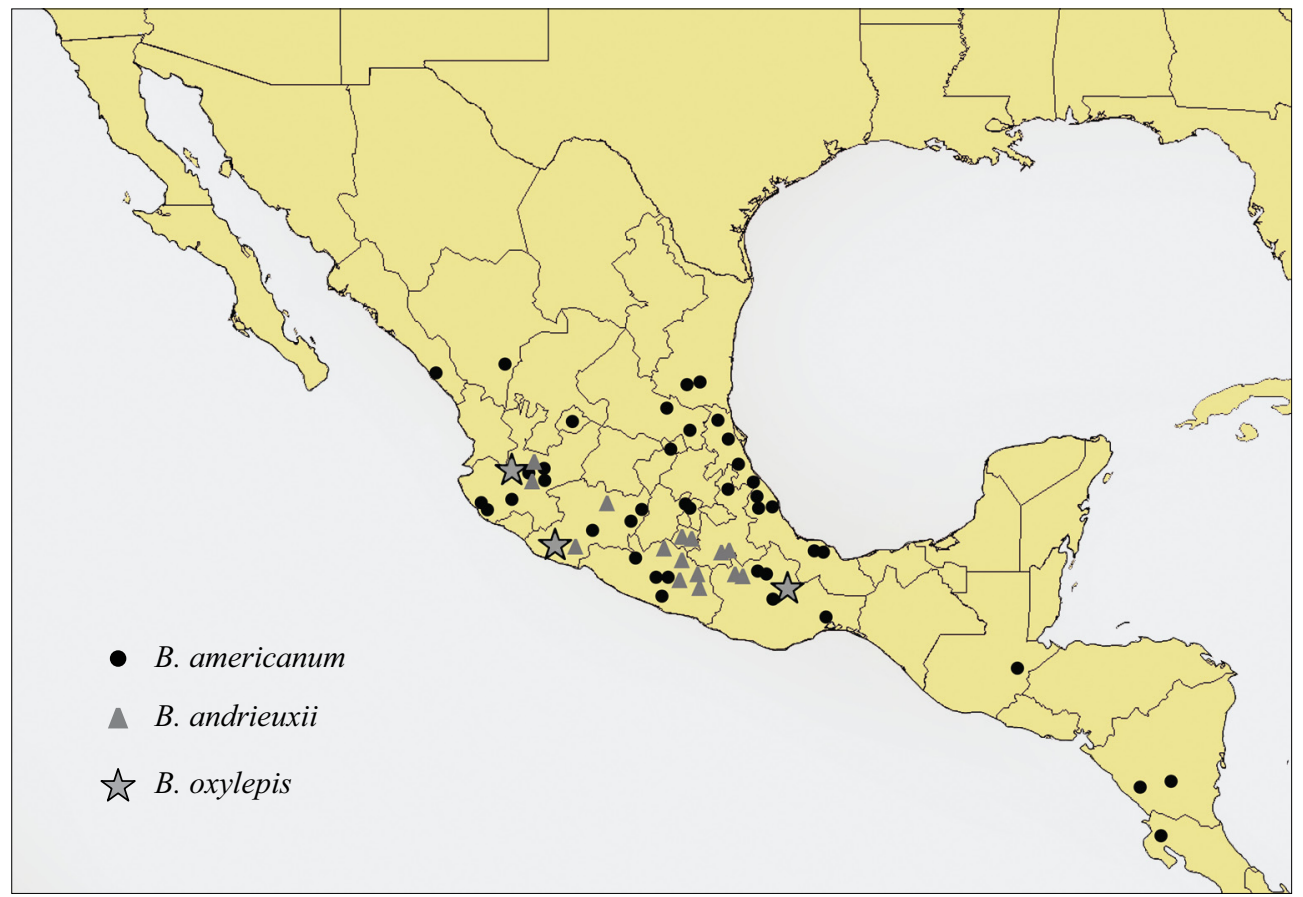

Fig. 3. Mapa de la distribución de Bdallophytum americanum, B. andrieuxii y B. oxylepis. 
dispuestas de forma anular en el ápice de la columna, conectivo apical 2-4.5 mm largo, acuminado. Flores femeninas con brácteas 5.5-9(-10) x 3.9-5.5 mm, ovadas o elípticas, margen serrado, pardas a negras; perianto 7-9-segmentado, (4.7-)5.8-11 $\mathrm{mm}$ alto, 4.5-8.5 mm diámetro en la base, de color guinda, estilos 3.5-6.5 mm largo, estigmas 9-10-lobulados, circulares a oblongos, amarillos. Bayas 1.2-1.5 cm diámetro, no concrescentes, pardas; semillas 0.4-0.5 mm largo.

Distribución y ecología. Especie que se registra desde el centro de México (en los estados de Aguascalientes, Durango, Guerrero, Jalisco, México, Michoacán, Oaxaca, Puebla, Querétaro, San Luis Potosí, Sinaloa, Tamaulipas y Veracruz) hasta Costa Rica (Gómez, 1983; Fig. 3). Este taxon se ha encontrado parasitando distintas especies de Bursera, aunque también puede encontrarse en Gyrocarpus americanus Jacq. (Moreno 17068 (MEXU)), Haematoxylum brasiletto H. Karst. (Pöll 5586 (MO foto de la colecta)), así como especies de Cochlospermum Kunth, Ficus L. y Guazuma Mill. (Gómez, 1983), en vegetación de bosque tropical caducifolio, bosque tropical perennifolio y subcaducifolio y también en matorral xerófilo, en elevaciones de 80 hasta $1840 \mathrm{~m}$ s.n.m. La floración ocurre principalmente de abril a agosto y la fructificación de agosto a noviembre.

Nombres comunes y usos. En Guerrero se le llama "flor de tierra" (Calónico 1099 (FCME)); en San Luis Potosí se le denomina "Boo'waat wits" (huasteco) y se le atribuyen ciertas propiedades medicinales (Alcorn 3252 (MEXU)).

Ejemplares examinados. GUATEMALA: Zacapa. Vicinity Pasabien, Pöll 5586 (MO foto de la colecta). MÉXICO: Aguascalientes. Mpio. Calvillo. Cañada los Adobes, de la Cerda 6690 (MEXU). Durango. Mpio. Mezquital. $10 \mathrm{~km} \mathrm{SW} \mathrm{de}$ Mezquital, Tenorio et al. 9597 (MEXU*). Guerrero. Mpio Eduardo Neri. La Yesera, $12.3 \mathrm{~km}$ al N de Zumpango del Río, Calónico 1099 (FCME); $10 \mathrm{~km}$ N de Zumpango del Río, sobre carr. a Iguala, Rzedowski 35812 (ENCB); Puente Mexcala, Halbinger s.n. (MEXU); Puente Mexcala, Matuda y Halbinger s.n. (MEXU); $83.5 \mathrm{~km}$ NE of Mex 200 jet in La Salitrera, on the way (Mex134) to Cd. Altamirano, Yahara et al. 2237 (MEXU). Jalisco. Mpio. Bolaños. Rancho El Gallinero, 15 km NE de Bolaños, antes de llegar al crucero de Bolaños, carr. V. Guerrero-San Martín, Flores et al. 1840 (XAL). Mpio. Jocotepec. Barranca del Huarache, enfrente (SE) de Zapotitán de Hidalgo, Machuca y Cházaro 7558 (XAL). Mpio. La Huerta. Estación de Biología Chamela, vereda Calandria, Magallanes 4401 (MEXU). Mpio. Tuxcacuesco. Cerro del Palacio, 5-6 km WSW de Tuxcacuesco, 5-6 km ENE de Zenzontla, Santana et al. 
6468 (MEXU, ZEA); Cerro del Palacio, 5-6 km WSW de Tuxcacuesco, Muñoz 6817 (MEXU, ZEA). Mpio. Zapopan. Cercanías del Puente Guadalupe, De Puga 7422 (ENCB); Barranca los Tempisques, cercana a la ex hacienda El Lazo, García-Larios y Puga s.n. (MEXU). Mpio. sin definir. La Coyota o La Cortina, Cuevas et al. 4656 (ZEA). México. Mpio. Tejupilco. Los Bejucos, Matuda et al. 31378 a , b, c (MEXU*). Michoacán. Mpio. La Huacana. Ca. 4 km (by road) SE of San Pedro Barajas, along Mex 37 W of El Limoncito, Steinmann 3080 (MEXU*). Mpio. Los Reyes. Barranca Los Chorros del Varal, García y Cházaro 3671 (XAL). Mpio. Tuzantla. 5 km S de Paso de la Tierra Caliente, González-Medrano et al. 4085 (MEXU). Oaxaca. Dto. Cuicatlán. Cerro Huarache Pintado, San José del Chilar, Cruz-Espinosa y San Pedro 1069 (MEXU*); cerro El Zacatal, 4 km S de San José del Chilar, Cruz-Espinosa et al. 1119 (MEXU); San Juan Bautista Cuicatlán, cerro El Zacatal, 4 km S de San José El Chilar, Cruz-Espinosa et al. 1182 (MEXU*); $1.5 \mathrm{~km}$ en línea recta, S de Santiago Dominguillo, carretera Oaxaca - San Juan Bautista Cuicatlán, Juárez-García et al. 776 (MEXU*); 5 km SE de San Juan Bautista Cuicatlán, desviación a San Pedro Jocotipac, González-Medrano et al. F-1538 (ENCB, MEXU*); 9 km NE de San Juan Bautista Cuicatlán, rumbo a Concepción Pápalo, González-Medrano et al. F-1700 (ENCB, MEXU*). Dto. Etla. El Progreso Sosola, camino real a La Calera (en el Cerro Buenavista), Salinas y Flores 7368 (MEXU*); Cerro Buenavista, ca. $1 \mathrm{~km} \mathrm{~N} \mathrm{de}$ El Parián, junto al río, Salinas y Martínez-Correa 6239a (MEXU*). Dto. Tehuantepec: Tehuantepec, Matuda 587 (MEXU). Puebla. Mpio. San José Miahuatlán. Cerro Petlanco, Salinas s.n. (MEXU*); El Ajengibre, Bravo s.n. (MEXU). Querétaro. Mpio. Arroyo Seco. 2-3 km N de Mesa de Agua Fría, Carranza 2194 (MEXU). San Luis Potosí. Mpio. Aquismón. Tancuime, Alcorn 3252 (MEXU). Mpio. Ciudad del Maíz. Chupaderos, Rzedowski 7751 (ENCB). Sinaloa. Mpio. Concordia. Paraje La Retumbadora, $5 \mathrm{~km} \mathrm{~S}$ de El Cuajote, Guizar 3306 (MEXU). Tamaulipas. Mpio. Gómez Farías. 2 km N de Gómez Farías, Díaz s.n. (FCME); E of Los Cedros station, on W facing slope, Seigler y Jones DS14039 (XAL); Aprox. 2 km E de Gómez Farías, Sierra Chiquita, Valiente y Viveros 228 (ENCB, MEXU, XAL); aprox. $3 \mathrm{~km} \mathrm{~W}$ de Gómez Farías, Valiente et al. 313 (MEXU); 12 km SSW del poblado Gómez Farías, Bocatoma. Cerca de la estación meteorológica Ahualulco de la SAHR, Valiente et al. 483 (MEXU). Veracruz. Mpio. Actopan. Cerro de La Mesa (Sierra de Manuel Díaz), Acosta y Calzada 955 (XAL); ejido Villa Rica, Acosta y Vázquez 638 (XAL); playa Cansa Burros, 2 km S de la Mancha, Acosta y Vázquez 789 (XAL); Villa Rica, cerca de Villa Rica, García 614 (XAL); Estación Biológica El Morro de la Mancha, Guerrero 1748 (XAL). Mpio. Dos Ríos. Cerro Gordo, Ventura 1502 (MEXU); La Cumbre, Ventura 4005 (ENCB). Mpio. Emiliano Zapata. 1/2 km de la desv. a Ca- 
rrizal, carr. Xalapa, Calzada 1829 (XAL). Mpio. La Antigua. El Rincón del Pirata, Ventura 14932 (ENCB, MEXU, XAL). Mpio. Martínez de la Torre. Ixtacuaco, Ventura 19674 (ENCB, MEXU*); Cañadas, Ventura 19798 (ENCB). Mpio. Ozuluama. Camino la Laja-Ozuluama, km 14, Chiang 99 (MEXU). Mpio. Pánuco. Cerro Topila, ejido Benito Juárez, Gutiérrez y Guerrero 1997 (MEXU*). Mpio. Papantla. 2 km delante de Papantla, Vázquez 1155 (XAL). Mpio. Paso de Ovejas. Camino a Acasónica, $2 \mathrm{~km}$ antes de Acasónica, Vázquez 575 (XAL). Mpio. Puente Nacional. Barranca de Pachuquilla, $2 \mathrm{~km}$ SW de dicha población, Medina y Vázquez 439 (MEXU, XAL). Mpio. San Andrés Tuxtla. N and E sides of Laguna Encantada, $3 \mathrm{~km}$ NE of San Andrés Tuxtla, Nee et al. 24751 (ENCB, MEXU, XAL); Laguna Encantada, ca. $3 \mathrm{~km}$ of Sn. Andrés Tuxtla, San Andrés Tuxtla, Dressler y Jones 137 (MEXU); E of Sn Andrés Tuxtla, Dressler y Jones 138 (MEXU). Mpio. Tepetzintlán. San José de Copaltitlan, 7 km NE de Tepetzintlán, Castillo y Benavides 2579 (XAL). Mpio. Yecuatla. La Independencia, orilla W del poblado, dirección Arroyo Burro, Gutiérrez 3767 (MEXU). Cerro Monte de Oro, Dorantes et al. 924 (MEXU*). NICARAGUA:

Chontales. Hda. Corpus, $2 \mathrm{~km} \mathrm{~S}$ of the hwy between El Guasimo and Juigalpa, Miller et al. 1057 (MEXU*); Hacienda Corpus, $11 \mathrm{~km} \mathrm{~W}$ of Juigalpa, Miller y Nee 1384 (ENCB, MEXU*); Hda. Corpus, W of Juigalpa, Stevens 21780 (MEXU*, MO foto de la colecta). Granada. Casa Tejas, $21 \mathrm{~km}$ de la carr. Nandaime-Granada, Moreno 17068 (MEXU).

Comentarios. La combinación Bdallophytum americanum (basada sobre Cytinus americanus) es comúnmente adjudicada a Harms (1935), pero no es legítima, ya que se trata de un homónimo posterior al propuesto por Solms-Laubach (1889), quien reconoce las especies $B$. andrieuxii y a $B$. americanum, por lo que cabe considerar a $B$. ceratantherum Eichler como sinónimo de este último.

El ejemplar tipo correspondiente a $B$. ceratantherum no fue encontrado en el herbario de Ginebra $(\mathrm{G})$, como se indica en el protólogo; posiblemente se encuentre en alguno de los herbarios donde Eichler trabajaba y no fue devuelto en su momento al herbario de De Candolle. El mencionado nombre se corrobora aquí como sinónimo de B. americanum, como ya habían considerado autores previos (Solms-Laubach, 1889; Harms, 1935; Kuijt, 2001), pues las características del androceo en B. ceratantherum, mencionadas en la descripción original, corresponden con las de B. americanum.

García-Franco y Rico-Gray (1997b) observaron en la región tropical de los Tuxtlas, Veracruz, que los frutos de B. americanum son consumidos por el roedor Peromyscus mexicanus mexicanus, pero dada la circunstancia de que la distribución de este roedor se restringe al eje neovolcánico (Ceballos, 2005), deben ser otros ma- 
míferos y/o insectos los que se encargan de la dispersión del parásito en los demás sectores de su área.

Bdallophytum andrieuxii Eichler, Bot. Zeitung (Berlin) 30: 715, t. 8. 1872; Cytinus andrieuxii (Eichler) Hemsl., Biol. Cent.-Amer., Bot. 3: 41. 1882; Hypocistis andrieuxii (Eichler) Kuntze, Revis. Gen. Pl. 2: 563. 1891; Scytanthus andrieuxii (Eichler) Solms in Engler, Pflanzenr. IV. 75(5): 18. 1901; Cytinus americanus Hook. f. in DC., Prodr. 17: 108. 1873. pro parte. TIPO: México. Oaxaca: in Sylvis Ditionis Oaxacanae, inter Huauapan (Huajuapam) et Oaxaca, jun 1834, G. Andrieux 50 (holotipo G-DC!; isotipo K!). (Fig. 2).

Hierbas 10-12.5 cm alto, dioicas; escamas 1.6-4.5(-4.8) x 1.4-3.8 mm, ovadas a lanceoladas, margen irregularmente serrado o entero, suculentas, pardas a moradas, glabras a pubescente-glandulares. Inflorescencias con más de 40 flores. Flores masculinas con brácteas (2.1-)4.2-6.6 x 1.3-3.8(5.6) mm, ovadas a espatuladas, margen serrado, moradas a negras; perianto 5-7-segmentado, 2.9-3.8 mm alto, 3.2-3.5 mm diámetro en la base, morado; columna estaminal 1-3 mm largo, anteras 5-14(-18), 1-2.1 mm largo, generalmente curvadas, dispuestas de forma irregular en el ápice de la columna, conectivo apical ausente. Flores femeninas con brácteas 3.3-5.5 x 1.8-4 mm, con margen serrado, moradas, pubescente-glandulares, periantos 5-7-segmentados, 3-4.3 $\mathrm{mm}$ alto, (2-)3-6.7(-8) $\mathrm{mm}$ diámetro en la base, morados, estilos 0.9-1.5 mm largo, estigmas 8-12-lobulados, oblongos, probablemente amarillos. Bayas 0.8-1.2 cm diámetro, concrescentes, pardas; semillas 0.3$0.4 \mathrm{~mm}$ largo.

Distribución y ecología. Especie endémica de México, presente en los estados de Guerrero, Michoacán, Morelos, Oaxaca y Puebla (Fig. 3). Este taxon se ha encontrado parasitando a Bursera aloexylon (Schiede ex Schltdl.) Engl. (Castro y Villegas 1136, Valenzuela 1 (HUMO)) y otras especies de Bursera, así como a Juniperus (Alvarado-Cárdenas y Sandoval s.n. (MEXU)), en bosque tropical caducifolio y matorral xerófilo, en elevaciones de 1173 a $1410 \mathrm{~m}$. La floración y fructificación está registrada en junio y julio.

Nombres comunes. En Guerrero y Michoacán se le llama "flor de tierra" $(\mathrm{Ca}$ lónico 1031 (FCME) y Armas s.n. (MEXU)) y en Morelos “mojón de gañán” (Castro y Villegas 1136 (HUMO)). 
Ejemplares examinados. MÉXICO: Guerrero: Mpio. Alpuyeca. $0.5 \mathrm{~km}$ al E de Tecoyo, Calónico 408 (FCME); $0.5 \mathrm{~km}$ al SW de Tecoyo, Calónico 1810 (FCME). Mpio. Eduardo Neri. Amatitlán, Calónico 1031 (FCME); Axaxacoalco, 1 km al W, Valencia 1193 (FCME), 1194 (FCME); cañada del Zopilote, Bravo s.n. (MEXU*). Mpio. Huamuxtitlán. $6 \mathrm{~km}$ (15 grados) SE de Huamuxtitlán hacia Cualac, Limón 74-III (FCME); 6 km (15 grados) SE de Huamuxtitlán hacia Tlaquiltepec, Soto 658 (FCME). Mpio. Mina. Trincheras, Hinton et al. 10134 (G); Mina, sin colector s.n. (MEXU*). Mpio. Xochihuehuetlán. Paraje Ocotepec, $2.75 \mathrm{~km}$ NNW de Jilotepec, Cerro Xilotzin, Moreno 956 (FCME). Mpio. Zicapa. $8 \mathrm{~km}$ al N de Zicapa, Monzon s.n. (FCME). Jalisco: Mpio. Guadalajara. Barranca de Huentitán El Alto, parte alta de los alrededores del templo, Macías 896 (ENCB). Mpio. Zapopan. W del Cerro El Mexicano, Barranca los Tempisques, cercana a la exhacienda El Lazo, García-Larios s.n. (ENCB). Michoacán: Mpio. Apatzingán. Apatzingán, Hinton 12012 (ENCB). Mpio. Arteaga, Potrero para el ganado, Armas s.n. (ENCB, MEXU*, XAL); potrero El Puerto, Armas s.n. (ENCB). Morelos: Mpio. Amacuzac. El Cristo, $10 \mathrm{~km} \mathrm{~N}$ de los límites estatales, entre Morelos y Guerrero, García-Mendoza et al. 6527 (MEXU). Mpio. Puente Ixtla. A $1 \mathrm{~km}$ al S de Tilzapotla, Juárez y Bustamante 1039 (HUMO). Mpio. Tlalquiltenango. A $7 \mathrm{~km}$ al NW de Huautla, Castro y Villegas 1136 (HUMO); $5 \mathrm{~km}$ de Xochipala, Cerros et al. 1065 (HUMO); 1071 (HUMO); Tlalquiltenango, Miranda 1483 (MEXU*); El Limón de Cuachichinola, Valenzuela 1 (HUMO). Oaxaca: Dto. Huajuapam: terracería entre Tultitlán de Guadalcázar y San Miguel Ixtapa, Guizar y Herrera 4065 (MEXU*); in Sylvis Ditionis Oaxacanae, inter Huauapan (Huajuapam) et Oaxaca, Andrieux 50 (G, K*). Puebla: Mpio. Caltepec. Cerros a $1 \mathrm{~km}$ W del poblado de San Juan Acatitlán, Alvarado-Cárdenas y Sandoval s.n. (MEXU*). Mpio. Izúcar de Matamoros. Entrada a la estación de M.O. Raboso, aprox. 9 km S de Raboso, carr. I. De Matamoros-Huajuapan de León, Panero et al. 5762a (MEXU*).

Comentarios. Los individuos femeninos de esta especie han sido determinados erróneamente como Bdallophytum americanum, pero se pueden identificar fácilmente porque $B$. andrieuxii tiene un mayor número de flores y la concrescencia de los ovarios en la fructificación es muy evidente (Fig. 2).

Bdallophytum oxylepis (B. L. Rob.) Harms in Engler \& Prantl, Nat. Pflanzenfam. ed. 2, 16b: 281. 1935; Cytinus oxylepis B. L. Rob., Proc. Amer. Acad. Arts 29: 321. 1894. TIPO: México. Jalisco. Zapotlán, 13-27 May 1893, C. G. Pringle 4373 (holotipo GH; isotipos MEXU!, MO foto!, NY foto!, US foto!) (Fig. 2). 
Hierbas 6.1-11 cm alto, polígamo-monoicas; escamas 2.3-4.5 x 2-2.2 mm, ovadas, margen irregularmente serrado a crenado, crasas, pardas a moradas, pubescente-glandulares. Inflorescencias con más de 40 flores. Flores femeninas con brácteas 2.3-4 x 1.5-2.0 mm, con margen serrado, moradas, pubescente-glandulares, periantos 5-7-segmentados, 2.5-3 mm alto, 2.6-3.5 mm diámetro en la base, morados, estilos 1-1.6 mm largo, estigmas 8-12-lobulados, oblongos, probablemente amarillos. Flores hermafroditas con brácteas (1.6-)3.3-4 x 1.5-2.4 mm, ovadas, con margen serrado, moradas a negras, pubescente-glandulares, perianto 5-7-segmentado, 2.7-3 mm alto, 2.6$3.5 \mathrm{~mm}$ diámetro en la base, morados; columna estaminal 1.1-1.6 mm largo, anteras (2-)4-7(-10), 1.1-1.6 mm largo, generalmente curvadas, dispuestas de forma anular y opuestas al estigma en el ápice de la columna, conectivo apical ausente; estilos 1.1-1.6 mm largo, estigmas 8-12-lobulados, oblongos, probablemente amarillos. Bayas 5.5-7.5 mm largo, mm diámetro, ovoides, concrescentes, pardas; semillas 0.3-0.4 mm largo.

Distribución y ecología. Especie endémica de México recolectada únicamente en los estados de Jalisco, Michoacán y Oaxaca. (Fig. 3). El taxon sólo se ha encontrado parasitando especies de Bursera, en vegetación de bosque tropical caducifolio y matorral xerófilo, en elevaciones de 950 a 1200 m s.n.m. La floración y fructificación ocurre de mayo a agosto.

Ejemplares examinados. MÉXICO: Jalisco: Mpio. Ciudad Guzmán. Zapotlán, Pringle 4373 (MEXU*). Michoacán: Mpio. Coalcomán. Salitre, Rzedowski 16712 (ENCB, MEXU*). Oaxaca: Mpio. sin definir. 5 km NW de Santa María Laxizanace, Reyes 1901 (MEXU*).

Comentario. Esta especie es muy parecida a Bdallophytum andrieuxii, ya que las anteras no tienen conectivo apical y los frutos son concrescentes, pero es fácil de separar este último por ser el único representante del género con flores hermafroditas y unisexuales femeninas en el mismo individuo.

\section{CONCLUSIONES}

El patrón de ordenación obtenido del análisis de coordenadas realizado para los ejemplares del género Bdallophytum permitió reconocer tres especies $B$. americanum, $B$. andrieuxii y $B$. oxylepis (Fig. 2). La subordinación de B. bambusarum y $B$. ceratantherum a sinónimos de $B$. americanum propuesta por algunos autores 
(Solms-Laubach, 1889; Kuijt, 2001; Carranza-González, 2002) se corrobora en este análisis (Fig. 1). Por otra parte, Gómez (1983) consideró este género como monotípico, reconociendo sólo a $B$. americanum y mencionando que las especies descritas no presentaban características relevantes para reconocerlas como entidades independientes. No obstante, es probable que Gómez (1983) no observara material de los demás componentes del género en otras colecciones, ya que existen diferencias evidentes no sólo en la talla de las inflorescencias y la concrescencia de los frutos. De acuerdo con este análisis, las principales discrepancias entre las especies radican en la disposición y forma de las anteras, el número de flores por inflorescencia, la presencia de flores unisexuales o hermafroditas y la longitud y color del perianto.

La evidencia encontrada hasta el momento permite resolver la problemática sobre la identidad y número de entidades en este género. Sin embargo, aún falta trabajo por hacer en el ámbito filogenético, donde se incluyan a todas las especies de Bdallophytum y de su grupo hermano Cytinus que nos permita generar hipótesis más robustas de las relaciones de estos taxones.

\section{AGRADECIMIENTOS}

Se agradece al Dr. V. Steinmann (Instituto de Ecología A.C.), a la Biól. R. Medina y a la M. en C. P. Hernández (Instituto de Biología de la UNAM), así como a dos revisores anónimos por los atinados comentarios en mejora de este trabajo; a la M. en C. V. Juárez-Jaimes (Instituto de Biología de la UNAM) por su ayuda en la solicitud del material tipo a los distintos herbarios; al Dr. E. Carranza (Instituto de Ecología A.C.) por facilitarme las imágenes digitales de Bdallophytum americanum; al P. de Biól. L. Galván (Universidad Autónoma del Estado de Morelos) por proveerme de información adicional de los ejemplares del HUMO y al M. en C. E. Domínguez-Licona (Instituto de Biología de la UNAM) por su apoyo en la elaboración de los mapas.

\section{LITERATURA CITADA}

Barkman, T. J., S. H. Lim, K. M. Salleh y J. Nais. 2004. Mitochondrial DNA sequences reveal the photosynthetic relatives of Rafflesia, the world's largest flower. Proc. Natl. Acad. Sci. U.S.A. 101: 787-792.

Blarer, A., D. L. Nickrent, H. Bänziger, P. K. Endress y Y.-L. Qiu. 2000. Phylogenetic relationships among genera of the parasitic family Rafflesiaceae s.l. based on nuclear 
ITS and SSU rDNA, mitochondrial LSU and SSU rDNA, atpl, and matR sequences. Amer. J. Bot. (Suppl. 6) 87: 171.

Bouman, F. y W. Meijer. 1986. Comparative seed morphology in Rafflesiaceae. Acta Bot. Neerl. 35: 521.

Bouman, F. y W. Meijer. 1994. Comparative structure of ovules and seeds in Rafflesiaceae. Pl. Syst. Evol. 193: 187-212.

Brown, R. 1844. Description of the female flower and fruit of Rafflesia arnoldi, with remarks on its affinities; and an illustration of the structure of Hydnora africana. Trans. Linn. Soc. Lond. 19: 221-238.

Burgoyne, P. M. 2006. A new species of Cytinus (Cytinaceae) from South Africa and Swaziland, with a key to the southern African species. Novon 16: 315-319.

Carranza-González, E. 2002. Rafflesiaceae. Flora del Bajío y de Regiones Adyacentes 107: $1-9$.

Ceballos, G. 2005. Peromyscus mexicanus. In: Ceballos, G. y G. Oliva (coord.). Los mamíferos silvestres de México. Comisión Nacional para el Estudio de la Biodiversidad, Fondo de Cultura Económica. México, D.F. pp. 758-759.

Cronquist, A. 1981. An integrated system of classification of flowering plants. Columbia University Press. New York. pp. 696-704.

Davis, C. C., M. Latvis, D. L. Nickrent, K. J. Wurdack y D. A. Baum. 2007. The evolution of floral gigantism in Rafflesiaceae. Science 315: 1812.

Eichler, A. W. 1872. Abermals ein neues Balanophoreen-Geschlecht. Bot. Zeitung (Berlin) 30: 709-715.

Endress, P. K. 1996. Diversity and evolutionary trends in angiosperms anthers. In: D'Arcy, W. G. y R. C. Keating (eds.). The anther, form, function and phylogeny. Cambridge University Press. New York. pp. 92-110.

García-Franco, J. G. 1996. Distribution and host specificity in the holoparasite Bdallophyton bambusarum (Rafflesiacease) in a tropical deciduous forest in Veracruz, Mexico. Biotropica 28: 759-762.

García-Franco, J. G. y V. Rico-Gray. 1997a. Dispersión, viabilidad, germinación y banco de semillas de Bdallophyton bambusarum (Rafflesiaceae) en la costa de Veracruz, México. Rev. Biol. Trop. 44: 87-94.

García-Franco, J. G. y V. Rico-Gray. 1997b. Reproductive biology of the holoparasitic endophyte Bdallophyton bambusarum (Rafflesiaceae). Bot. J. Linn. Soc. 123: 237247.

García-Franco, J., V. Sousa, L. Eguiarte y V. Rico-Gray. 1998. Genetic variation, genetic structure and effective population size in the tropical holoparasitic endophyte Bdallophyton bambusarum (Rafflesiaceae). Pl. Syst. Evol. 210: 271-288.

Gentry, A. H. 1973. Rafflesiaceae. In: Woodson, R. E. y R. W. Schery (eds.). Flora of Panama. Ann. Missouri Bot. Gard. 60(1): 17-21.

Gómez, L. D. 1983. Rafflesiaceae. Flora Costaricensis, Fieldiana Bot., New Series 13: 89-93.

Harms, H. 1935. Rafflesiaceae. In: Engler, A. y K. Prantl (eds.). Die natürlichen Pflanzenfamilien. ed. 2. Vol. 16b. Verlag von Wilhelm Engelmann. Leipzig. pp. 242-281.

Hemsley, W. B. 1882. Cytinaceae. In: Godman, F. D. y O. Salvin (eds.). Biologia Centrali Americana. Botany. Vol. 3. R. H. Porter. Londres. pp. 40-41. 
Hutchinson, J. 1959. The families of flowering plants. Clarendon Press. Oxford. 510 pp.

Hooker, J. D. 1873. Cytinaceae. In: Candolle, A. P. de (ed.). Prodrumus Systematis Naturalis Regni Vegetabilis. Vol. 17. G. Masson. Paris. pp. 106-116.

Kuijt, J. 1969. Rafflesiaceae, Hydnoraceae, and Balanophoraceae. In: Kuijt, J. (ed.). The biology of parasitic flowering plants. University of California Press. Berkeley. pp. 104-135.

Kuijt, J. 2001. Rafflesiaceae. In: Stevens, W. D., C. Ulloa Ulloa, A. Pool y O. M. Montiel (eds.). Flora de Nicaragua. Monogr. Syst. Bot. Missouri Bot. Gard. 85(3): 2189-2190.

Kuntze, C. E. O. 1891. Hypocistis, Scytanthus. In: Revisio generum plantarum. Vol. 2. Arthur Felix. Leipzig. p. 293.

Meijer, W. 1997. Rafflesiaceae. Flora Malesiana ser. 1. 13: 1-42.

Nickrent, D. L. 1997. Onwards. The Parasitic Plant Connection. http:/www.parasiticplants. siu.edu/UsingPPC.html

Nickrent, D. L. 2007. Cytinaceae are sister to Muntingiaceae. Taxon 56(4): 1129-1135.

Nickrent, D. L., A. Blarer, Y.-L. Qiu, R. Vidal-Russell y F. E. Anderson. 2004. Phylogenetic inference in Rafflesiales: the influence of rate heterogeneity and horizontal gene transfer. BMC. Evol. Biol. 4: 40.

Rohlf, F. 2004. NTSYS-pc:numerical taxonomy and multivariate analysis system, version 2.11T. New York, Exeter Software.

Solms-Laubach, H. 1889. Rafflesiaceae. In: Engler, A. y K. Prantl (eds.). Die natürlichen Pflanzenfamilien. Vol. 3(1). Verlag von Wilhelm Engelmann. Leipzig. pp. 224-282.

Solms-Laubach, H. 1901. Rafflesiaceae. In: Engler, A. y K. Prantl (eds.). Das Pflanzenreich. Vol. IV. 75. Verlag von Wilhelm Engelmann. Leipzig. pp. 1-19.

Stevens, P. F. (2001 onwards). Angiosperm phylogeny website. Version 6, May 2005 (and more or less continuously updated since). http://www.mobot.org/MOBOT/research/ APweb/

Takhtajan, A. 1997. Diversity and classification of flowering plants. Columbia University Press. New York. pp. 66-69. 
